\title{
Helminthes parasites des Micromammifères des Pyrénées-Orientales
}

\author{
II. Les Plathelminthes de Soricinae \\ par Joseph JOURDANE \\ (Collaboration technique M.-Th. Almeras) \\ Département de Biologie animale, Centre Universitaire, F.66-Perpignan
}

\begin{abstract}
Résumê
L'auteur donne l'inventaire des Plathelminthes rencontrés chez les Soricinae (Neomys fodiens, Sorex araneus, Sorex minutus) dans l'Est des Pyrénées. Plusieurs espèces sont nouvelles pour la faune française. Ce sont: Hymenolepis diaphana Cholodkowsky, 1906 ; Hymenolepis globosoides (Soltys, 1954) ; Hymenolepis jacutensis (Spassky et Morosov, 1959); Hymenolepis stefanskii Zarnowsky, 1954 et Nephrotrema truncatum (Leuckart, 1842). Les Cyclophyllides Hymenolepis alpestris Baer, 1931 et Hymenolepis jacutensis sont redécrits. Les espèces Brachylaemus oesophagei Schaldybin, 1953 et Soricitrema baeri Bychovskaya-Pavlovskaya et al., 1970 sont mises en synonymie respectivement avec Brachylaemus fulvus Dujardin, 1843 et Nephrotrema truncatum (Leuckart, 1842). Le Digène Pseudoleucochloridium soricis (Soltys, 1952) est considéré par l'auteur comme un parasite d'Oiseau.
\end{abstract}

\section{Summary}

The author gives the inventory of the Plathelminthes found on Soricinae (Neomys fodiens, Sorex araneus and Sorex minutus) in the eastern Pyrenées. Several species are new in French fauna: Hymenolepis diaphana Cholodkowsky, 1906; Hymenolepis globo. soides (Soltys, 1954); Hymenolepis jacutensis (Spassky et Morosov. 1959); Hymenolepis stefanskii Zarnowsky, 1954 et Nephrotrema

\footnotetext{
Avant de commencer cette étude, nous tenons à exprimer tous nos remerciements à $\mathrm{M}$. Claude Vaucher, Chef de Travaux à l'Université de Neuchâtel, qui nous a permis de voir ses préparations et qui nous a aimablement confirmé certaines de nos déterminations.
} 
truncatum (Leuckart, 1842). Two Cyclophyllidae are described Hymenolepis alpestris Baer, 1931 and Hymenolepis jacutensis. The Digenea Brachylaemus oesophagei Scholdybin, 1953 et Soricitrema baeri Bychowskaya-Pavlovskaya et al., 1970 are placed in synonym with Brachylaemus fulvus Dujardin, 1843 and Nephrotrema truncatum (Leuckart, 1842) respectively. The Trematoda Pseudoleucochloridium soricis (Soltys, 1952) is considered by the author as a bird'parasite.

Dans le cadre des recherches que nous poursuivons sur les cycles biologiques des Plathelminthes de Micromammifères pyrénéens, nous avons eu l'occasion, ces dernières années, de capturer un certain nombre de Mammifères Soricidés et d'en récolter les endoparasites. Dans un précédent travail, nous avons étudié les Cestodes de Neomys fodiens. Depuis, nous avons disséqué deux autres espèces de Soricidés et terminé l'étude systématique des Digènes de la Musaraigne aquatique, ce qui nous permet aujourd'hui de donner un inventaire helminthologique beaucoup plus complet des Insectivores Soricinés des Pyrénées-Orientales. Quelques espèces rencontrées étant encore fort mal connues, nous sommes amené à en préciser les descriptions.

Ayant pu récolter les œufs des parasites en vue de la réalisation expérimentale de leur cycle, nous donnons pour la plupart des espèces un dessin de l'œuf et ses dimensions mesurées sur le vivant, sans aplatissement.

L'inventaire helminthologique que nous présentons porte sur les hôtes suivants disséqués :

- 99 Neomys fodiens (Pennant).

- 26 Sorex minutus L.

- 18 Sorex araneus $L$.

Le tableau 1 montre la fréquence des parasites chez la Musaraigne aquatique, Neomys fodiens. Les Musaraignes ayant été capturées uniquement pendant la saison

\section{Tableau 1}

Pourcentages de Neomys fodiens parasitées pendant la saison estivale (juillet, août, septembre)

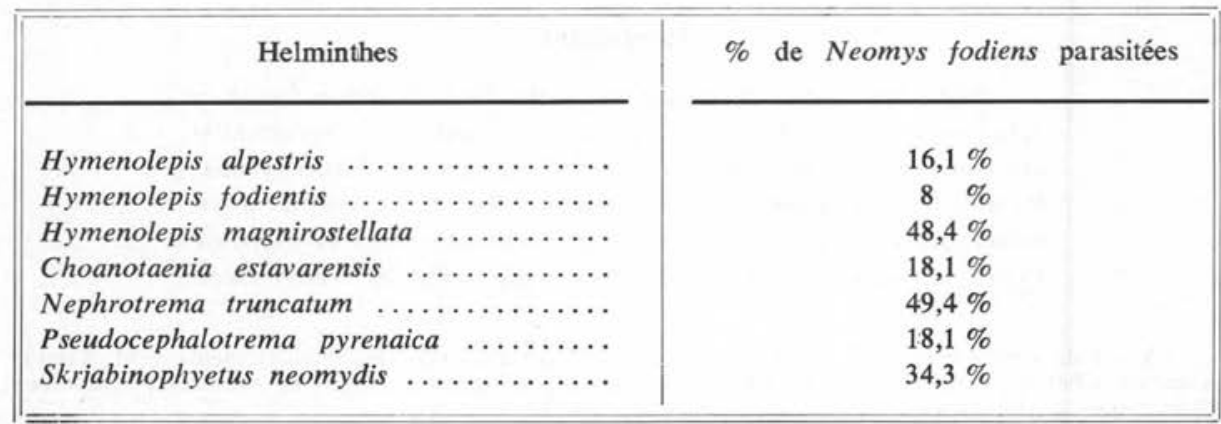


estivale, ces fréquences ne doivent être considérées comme significatives que pour cette saison. L'échantillon de Musaraignes du genre Sorex étudiées jusqu'ici étant assez faible, nous ne donnons pas pour ces espèces les pourcentages de parasitisme. Le tableau 2 indique la parasitofaune des deux espèces de Sorex.

\section{Tableau 2}

Parasitofaune des Musaraignes du genre Sorex

\begin{tabular}{|c|c|c|}
\hline Helminthes & Sorex minutus & Sorex araneus \\
\hline Hymenolepis diaphana...$\ldots \ldots \ldots$ & + & + \\
\hline Hymenolepis furcata $\ldots \ldots \ldots \ldots \ldots$ & + & + \\
\hline Hymenolepis globosoides .......... & + & + \\
\hline Hymenolepis jacutensis .......... & + & + \\
\hline Hymenolepis prolifer ............ & + & + \\
\hline Hymenolepis schaldybini .......... & + & + \\
\hline Hymenolepis stefanskii $\ldots \ldots \ldots \ldots$ & + & + \\
\hline Hymenolepis scutigera $\ldots . \ldots \ldots \ldots$ & + & + \\
\hline Choanotaenia crassiscolex ......... & + & + \\
\hline Brachylaemus fulvus ............. & + & + \\
\hline Nephrotrema truncatum $\ldots \ldots \ldots$ & + & + \\
\hline Pseudoleucochloridium soricis ..... & - & + \\
\hline
\end{tabular}

\section{A. - PLATHELMINTHES PARASITES DE NEOMYS FODIENS}

\section{Cestodes cyclophyllides}

Hymenolepis alpestris, Baer, 1931.

Cette espèce, décrite en Suisse, ne semble plus avoir été signalée depuis en Europe (1). Nous donnons ci-après une description de cette espèce rare.

Les individus mesurent de 8 à $25 \mathrm{~mm}$ de longueur. Les anneaux sexuellement mûrs mesurent en moyenne $180 \mu$ de longueur sur $240 \mu$ de largeur (fig. $1 \mathrm{~A}$ et 2 ). Nous avons compté en moyenne 200 segments par ver. Le scolex, sphérique, inerme, mesure $240 \mu$ de diamètre en moyenne. Il ne montre pas de rostre différencié. Les ventouses, cotylédonnaires, mesurent en moyenne 84 sur $132 \mu$.

(1) La détermination de cette espèce a été faite après comparaison avec les types déposés à l'Université de Neuchâtel. 
SyStème osmo-RÉgulateur : Il comprend quatre vaisseaux, de diamètre différent : les deux ventraux ont une lumière très large $(16 \mu)$, les deux dorsaux sont plus fins ( $9 \mu$ de diamètre).

SYSTÈME GÉNITAL: Les pores génitaux sont unilatéraux et débouchent au tiers antérieur de chaque anneau. L'atrium génital, peu profond, mesure $12 \mu$ de longueur.
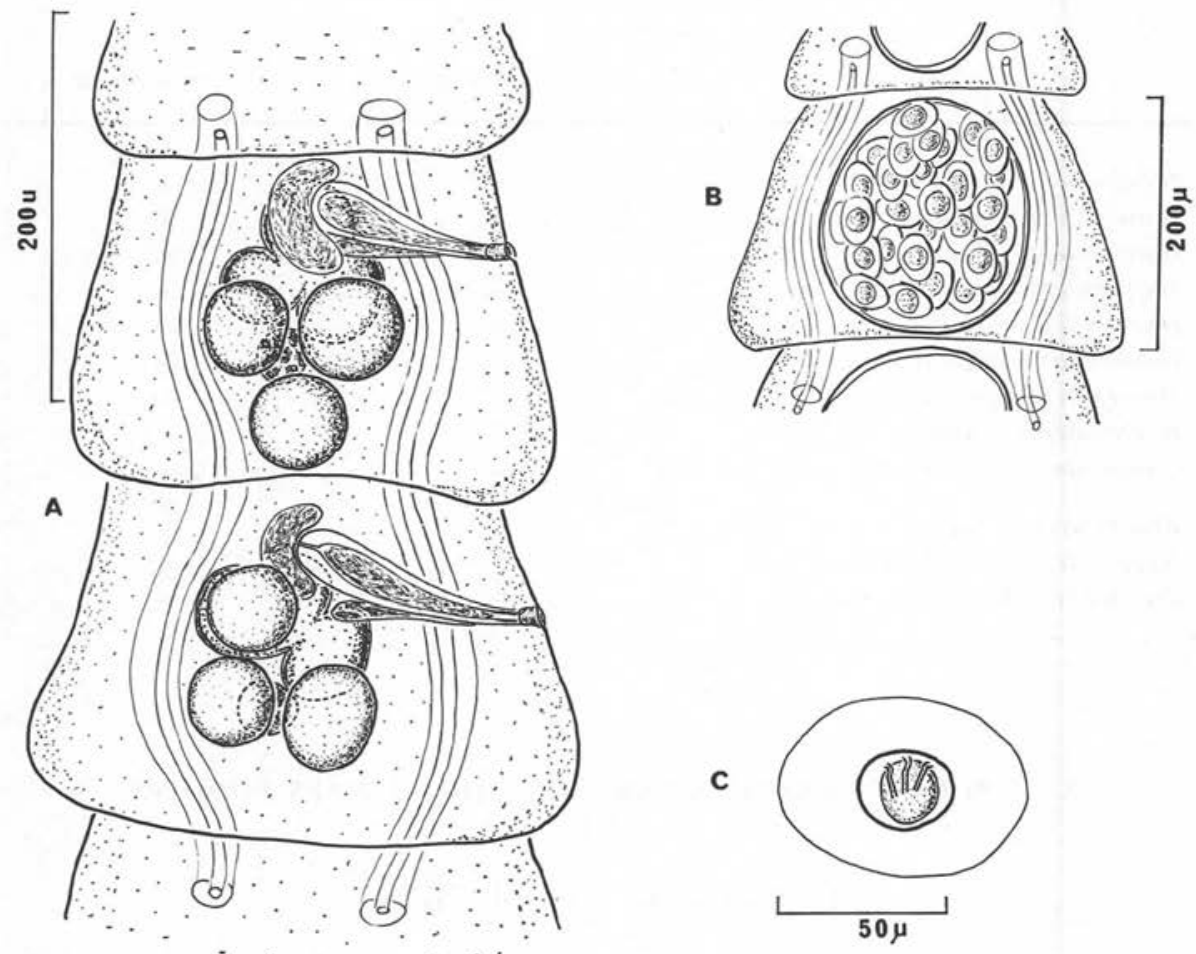

FIG. 1. Hymenolepis alpestris Baer. 1931. A : Proglottis mûrs: vue dorsale. B : Proglottis gravide: vue dorsale. C: Euf mûr

OrganES MÂles : Les trois testicules, contigus, toujours disposés en triangle, occupent la partie ventrale du segment. Ils sont sphériques et mesurent $60 \mu$ de diamètre en moyenne. De chaque testicule part un court canal efférent. La confluence des trois canaux efférents, au centre du segment, forme le canal déférent, qui remonte vers l'avant et débouche dans le lobe inférieur d'une curieuse vésicule séminale externe. Cette dernière à la forme d'un haricot $(55$ sur $25 \mu)$, dont le hile coiffe la partie proximale de la poche du cirre. 
La poche du cirre mesure $108 \mu$ de longueur et $20 \mu$ de largeur dans sa partie proximale qui renferme la vésicule séminale interne.

Le cirre évaginé mesure $40 \mu$ de longueur. Il est couvert d'épines très réduites.

ORganes femelles: L'ovaire, formé de trois lobes ventraux, est le plus souvent situé sous la masse testiculaire. Les lobes de l'ovaire, subsphériques, mesurent en moyenne $50 \mu$ de diamètre.

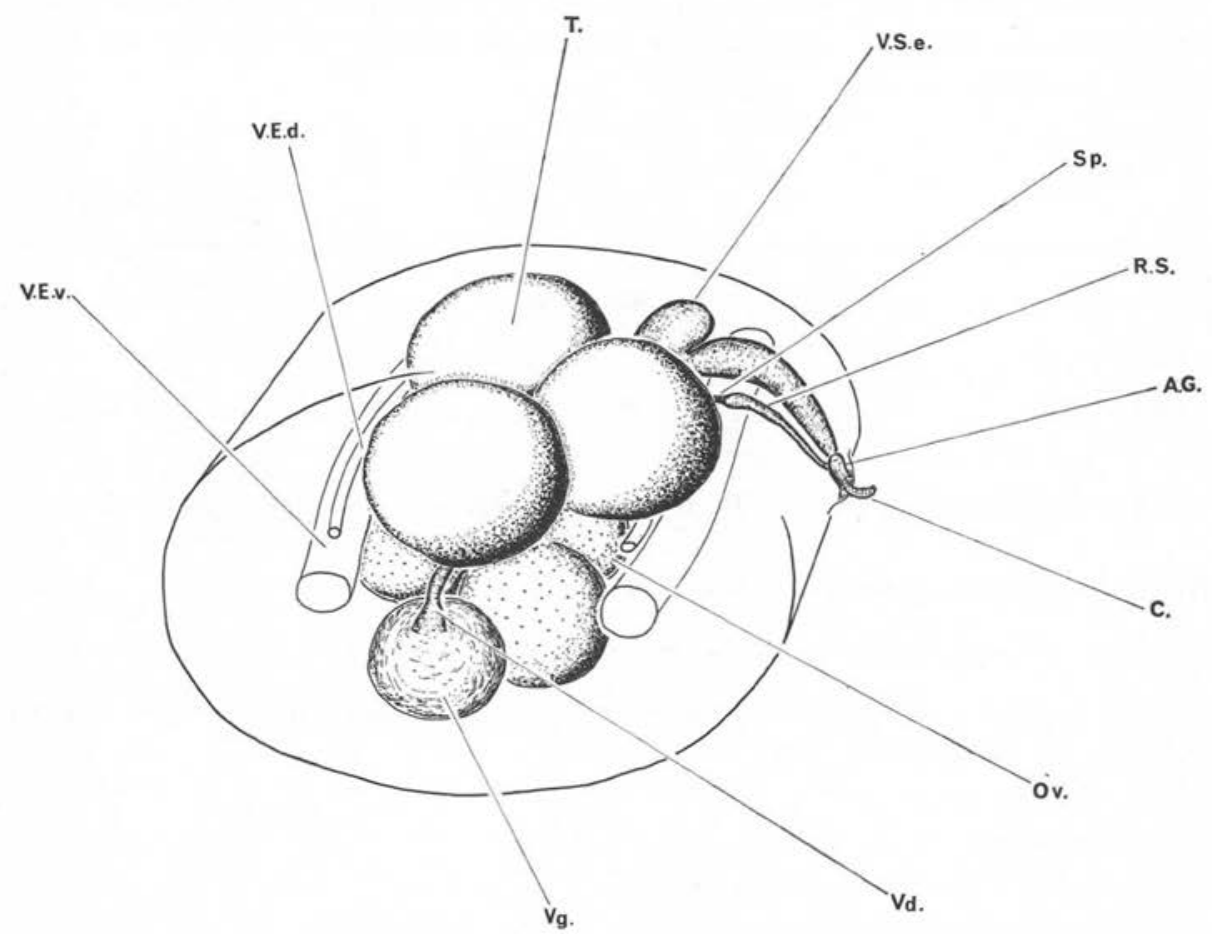

Fig. 2. - Hymenolepis alpestris Baer, 1931. Interprétation en vue cavalière de l'appareil génital d'un segment sexué. A.G. : Atrium génital ; C : cirre; Ov. : ovaire; R.S. : réceptacle séminal ; Sp. : sphincter ; T. : testicule ; V.E.d. : vaisseau excréteur dorsal ; V.E.v. : vaisseau excréteur ventral ; V.S.e.: vésicule séminale externe; Vd.: vitelloducte; Vg.: vitellogène

Le vagin se présente comme un long canal, légèrement dilaté dans sa région proximale qui joue le rôle de réceptacle séminal.

La glande vitellogène est située ventralement, en arrière de l'ovaire. Elle a la forme d'une sphère de $36 \mu$ de diamètre en moyenne.

Dans les anneaux mûrs, l'utérus, ovoïde (150 $\mu$ de diamètre en moyenne), ne s'étend jamais latéralement au-delà des canaux excréteurs (fig. 1 B).

L'embryophore mesure en moyenne 35 sur $28 \mu$. Les crochets de l'embryon hexacanthe ont $13 \mu$ de longueur (fig. 1 C). 
Hymenolepis fodientis, Vaucher, 1970.

Nous avons rapporté, dans un précédent travail, les grands Hymenolepis inermes rencontrés chez Neomys fodiens à l'espèce Hymenolepis globosoides (Soltys, 1954), parasite des Sorex en Europe. Vaucher (1970) a montré que les individus inermes de grande taille parasitant les Neomys appartenaient en réalité à une espèce nouvelle, qu'il a nommée $H$. fodientis. Après nouvel examen de nos préparations, nos observations sont en accord avec celles de cet auteur. Les grands Hymenolepis de Neomys se différencient en effet très nettement de l'espèce Hymenolepis globosoides des Sorex par les caractères suivants :

\begin{tabular}{|c|c|c|}
\hline & $\begin{array}{c}\text { H. Globosoides } \\
\text { (Soltys, 1954) }\end{array}$ & $\begin{array}{l}\text { H. Fodientis } \\
\text { Vaucher, } 1970\end{array}$ \\
\hline Taille du scolex $\ldots \ldots \ldots \ldots$ & 400 à $700 \mu$ & $300 \mu$ en moyenne \\
\hline Longueur du cirre évaginé ... & $110 \mu$ en moyenne & $25-30 \mu$ \\
\hline Embryophore $\quad \ldots \ldots \ldots \ldots \ldots$ & $38 \operatorname{sur} 16 \mu$ & $30 \operatorname{sur} 20 \mu$ \\
\hline
\end{tabular}

La morphologie de l'œuf de $H$. fodientis est représentée dans la figure $6 \mathrm{~A}$.

Hymenolepis magnirostellata, Baer, 1931.

C'est le Cyclophyllide le plus abondant ohez les Neomys de notre région.

Les moyennes des mesures faites sur les œufs provenant d'un abondant matériel frais sont les suivantes (fig. 6 B) :

- Euf $(90$ sur $53 \mu)$;

- Oncosphère (76 sur $30 \mu)$;

- Embryon hexacanthe (37 sur $26 \mu$ );

- Crochets $(14 \mu)$.

Choanotaenia estavarensis, Euzet et Jourdane, 1968.

Ce Cyclophyllide (pl. $1 \mathrm{E}$ et pl. 2) n'a été rencontré que chez la Musaraigne aquatique (Neomys fodiens). Nous avons donné dans un précédent travail la diagnose de l'espèce et les différences morphologiques et anatomiques qui la séparent de l'espèce Ch. crassiscolex. La récolte d'un abondant matériel nous a permis de mettre en évidence deux autres caractères constants chez l'espèce :

- Le strobile est en moyenne deux fois plus long que celui de Ch. crassiscolex.

- Le rostre est toujours trois fois plus long que celui de Ch. crassiscolex.

La morphologie des crochets et de l'œuf est donnée respectivement dans la planche 1 et la figure $6 \mathrm{C}$. 

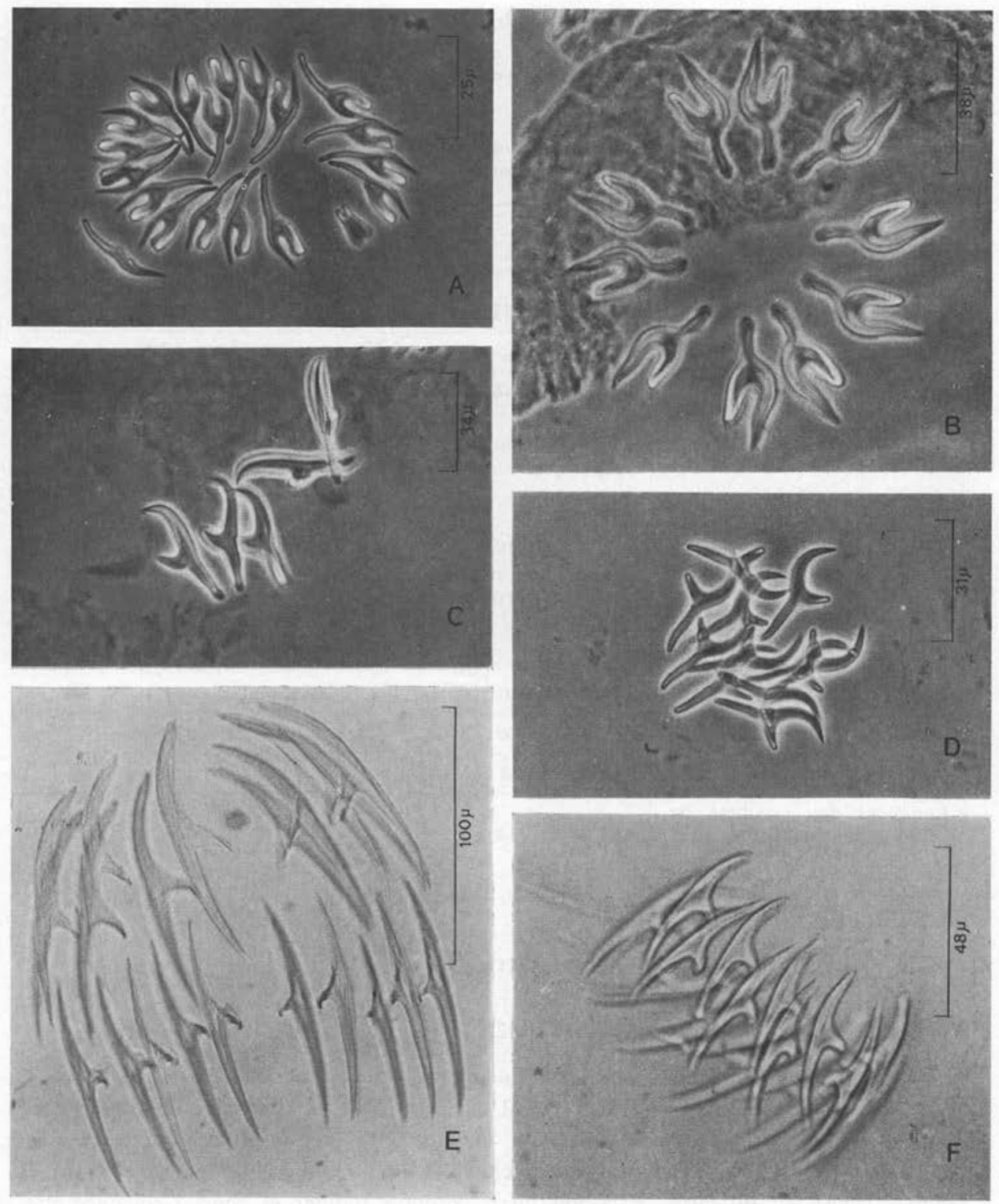

Planche 1. - Préparations au liquide de Berlese de crochets de Cyclophyllides de Soricinae. A : H. furcata; B: H. schaldybini; C: H. scutigera; D: H. stefanskii; E: Ch. estavarensis; F : Ch. crassiscolex 


\section{Tableau 3}

Mensurations des deux espèces de Choanotaenia (Les valeurs données représentent des moyennes)

\begin{tabular}{|c|c|c|}
\hline & $\begin{array}{c}\text { Choanotaenia crassiscolex } \\
\text { (Von Linstow, 1890) }\end{array}$ & $\begin{array}{l}\text { Choanotaenia estavarensis } \\
\text { (Euzet et Jou:dane, 1968) }\end{array}$ \\
\hline Longueur du ver $\ldots \ldots \ldots$ & $5,7 \mathrm{~mm}$ & $20 \mathrm{~mm}$ \\
\hline $\begin{array}{l}\text { Largeur du ver au niveau } \\
\text { des segments sexués } \ldots . . .\end{array}$ & $582 \mu$ & $778 \mu$ \\
\hline Ventouses $\ldots . . . \ldots \ldots$. & $438 \times 192 \mu$ & $400 \times 200 \mu$ \\
\hline Longueur du rostre $\ldots \ldots$. & $546 \mu$ & $1334 \mu$ \\
\hline Nombre de crochets .... & $\begin{array}{l}18 \text { à } 20 \text { (moyenne }=20 \\
\text { répartis en } 2 \text { couronnes) }\end{array}$ & $\begin{array}{c}20 \text { à } 21 \\
\text { (moyenne }=20 \\
\text { répartis en } 2 \text { couronnes) }\end{array}$ \\
\hline Longueur des crochets ... & $\begin{array}{l}48 \mu \\
\text { pour les crochets des } \\
\text { deux couronnes }\end{array}$ & $\begin{array}{c}(92 \text { à } 105,8 ; \text { moyenne }=99) \\
\text { pour les crochets de la } \\
\text { couronne supérieure } \\
(87 \text { à } 96,6 ; \text { moyenne }=94) \\
\text { pour les crochets de la } \\
\text { couronne inférieure }\end{array}$ \\
\hline Nombre de testicules .... & $\begin{array}{c}15 \text { à } 20 \\
\text { (nombre moyen }=17 \text { ) }\end{array}$ & $\begin{array}{c}15 \text { à } 20 \\
\text { (nombre moyen }=17)\end{array}$ \\
\hline Guf $\quad \ldots \ldots \ldots \ldots \ldots \ldots$ & $56 \times 37 \mu$ & $58 \times 37 \mu$ \\
\hline Embryophore $\ldots \ldots \ldots \ldots$ & $39 \times 25 \mu$ & $43 \times 26 \mu$ \\
\hline $\begin{array}{l}\text { Longueur des crochets de } \\
\text { l'embryon hexacanthe } . . .\end{array}$ & $14 \mu$ & $14 \mu$ \\
\hline
\end{tabular}

\section{Trématodes digènes}

Nephrotrema truncatum (Leuckart, 1842).

Ce parasite rénal a été trouvé pour la première fois chez la Musaraigne d'eau. Il a été par la suite redécrit par Baer (1932 et 1943), qui l'a rencontré en Suisse chez Neomys fodiens et chez Talpa europaea. Depuis cette redescription, il a été trouvé par Mituch (1964), en Tchécoslovaquie, chez Neomys anomalus et par Dimitrova et Genov (1966), en Bulgarie, à la fois chez Neomys fodiens et chez Crocidura suaveolens.

Bychovskaya-Pavloskaya et al. (1970) signalent en Russie l'existence, dans le rein des Musaraignes du genre Sorex, d'un nouveau Digène qu'ils nomment Soricitrema baeri. 
Nous avons retrouvé dans les Pyrénées, à la fois chez Neomys fodiens, Sorex minutus et Sorex araneus, un parasite rénal qui ne présentait pas de différences anatomiques suivant l'hôte de récolte (fig. 3). Tous les caractères signalés par Baer dans sa

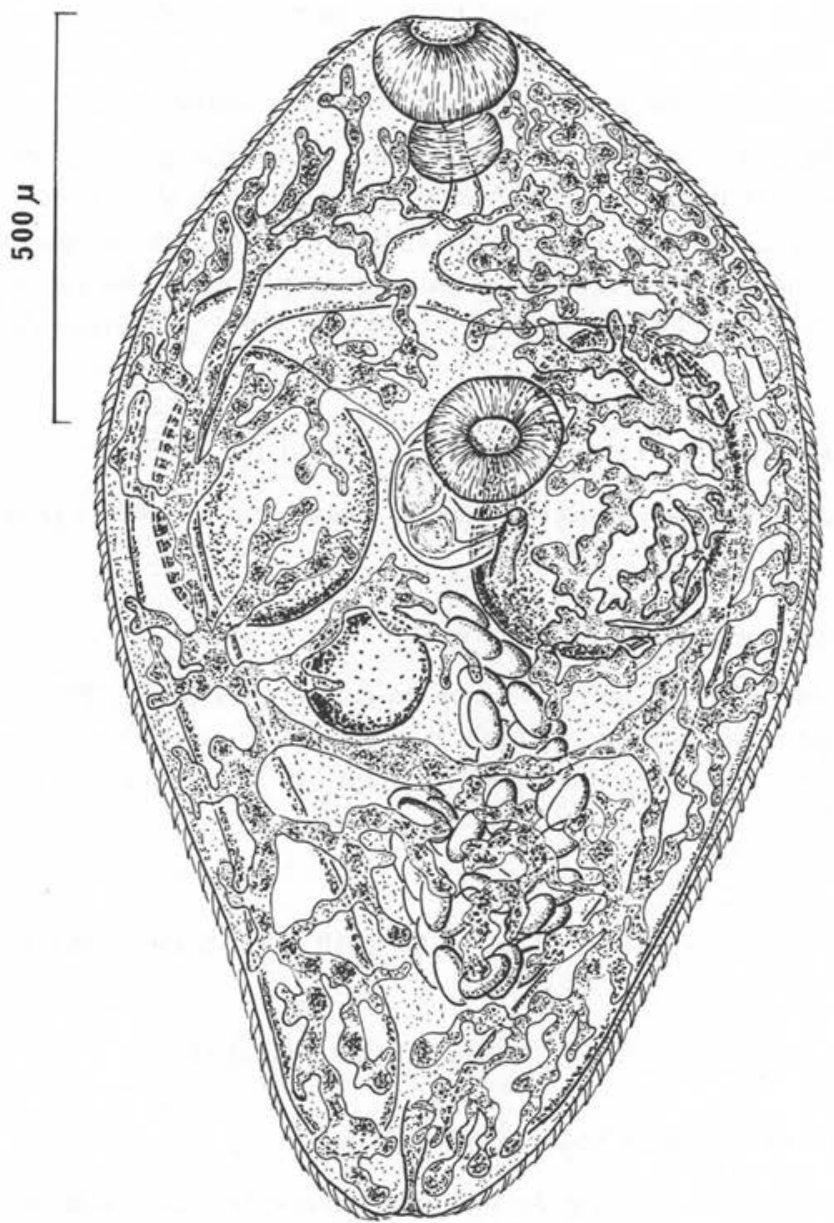

Fig. 3. - Nephrotrema truncatum (Leuckart, 1842). Digène mûr, vue ventrale.

redescription de Nephrotrema truncatum se retrouvent chez tous nos individus qui appartiennent donc tous à cette espèce.

Dans le schéma et les mensurations donnés par Bychovskaya-Pavlovskaya de l'espèce Soricitrema baeri, la seule différence avec la diagnose de Nephrotrema truncatum que nous ayons relevée concerne la taille des œufs. Les auteurs russes donnent 
des mensurations d'œufs $(78-83 \mu$ sur 44-49 $\mu)$ inférieures à celles observées par les auteurs : $90-108 \mu$ sur 54-61 $\mu$ pour Baer; 94 sur $49 \mu$ dans le présent travail. Ces auteurs motivent la création d'un nouveau genre en retenant comme caractères différentiels :

- Absence d'espace libre entre la bifurcation intestinale et l'acétabulum chez le genre Soricitrema.

- Plus grand développement de l'utérus chez ce dernier genre.

Nous admettons difficilement que les deux caractères ci-dessus indiqués puissent justifier la création d'une nouvelle espèce et, qui plus est, d'un nouveau genre.

Le peu de valeur systématique des caractères distinctifs retenus par les auteurs russes pour la création d'un nouveau genre ne nous permet pas de valider cette manière de voir et nous considérons Soricitrema baeri comme synonyme de Nephrotrema truncatum.

Pseudocephalotrema pyrenaica, Combes et Jourdane, 1970.

Cette espèce, récemment décrite, semble uniquement localisée à la région pyrénéenne.

Skrjabinophyetus neomydis, Dimitrova et Genov, 1967.

Nous avons déjà signalé la présence de ce Digène dans les Pyrénées. Ce parasite montre une aire très morcelée puisqu'il n'a été signalé jusqu'ici qu'en Bulgarie (Dimitrova et Genov, 1967), en Allemagne (Brendow, 1970) et dans les Pyrénées.

\section{B. - PLATHELMINTHES PARASITES DE SOREX}

\section{Cestodes cyclophyllides}

Hymenolepis diaphana, Cholodkowsky, 1906.

Ce Cyclophyllide est l'espèce que nous avons le plus fréquemment rencontrée chez les Musaraignes du genre Sorex. L'anatomie et les mensurations de nos échantillons s'accordent parfaitement avec celles données dans les descriptions très complètes de l'espèce faites par Kisielewska (1961) et Vaucher (1970).

Quelques précisions concernant la maturation des segments ovigères nous paraissent cependant utiles à ajouter. Vaucher tout comme Kisielewska mentionnent «une résorption des parois intersegmentaires, entraînant la réunion des œufs de 2 à 18 anneaux dans une enveloppe musculaire commune». Nous n'avons pu observer quant à nous ce phénomène de coalescence des anneaux ovigères, même sur les fragments 

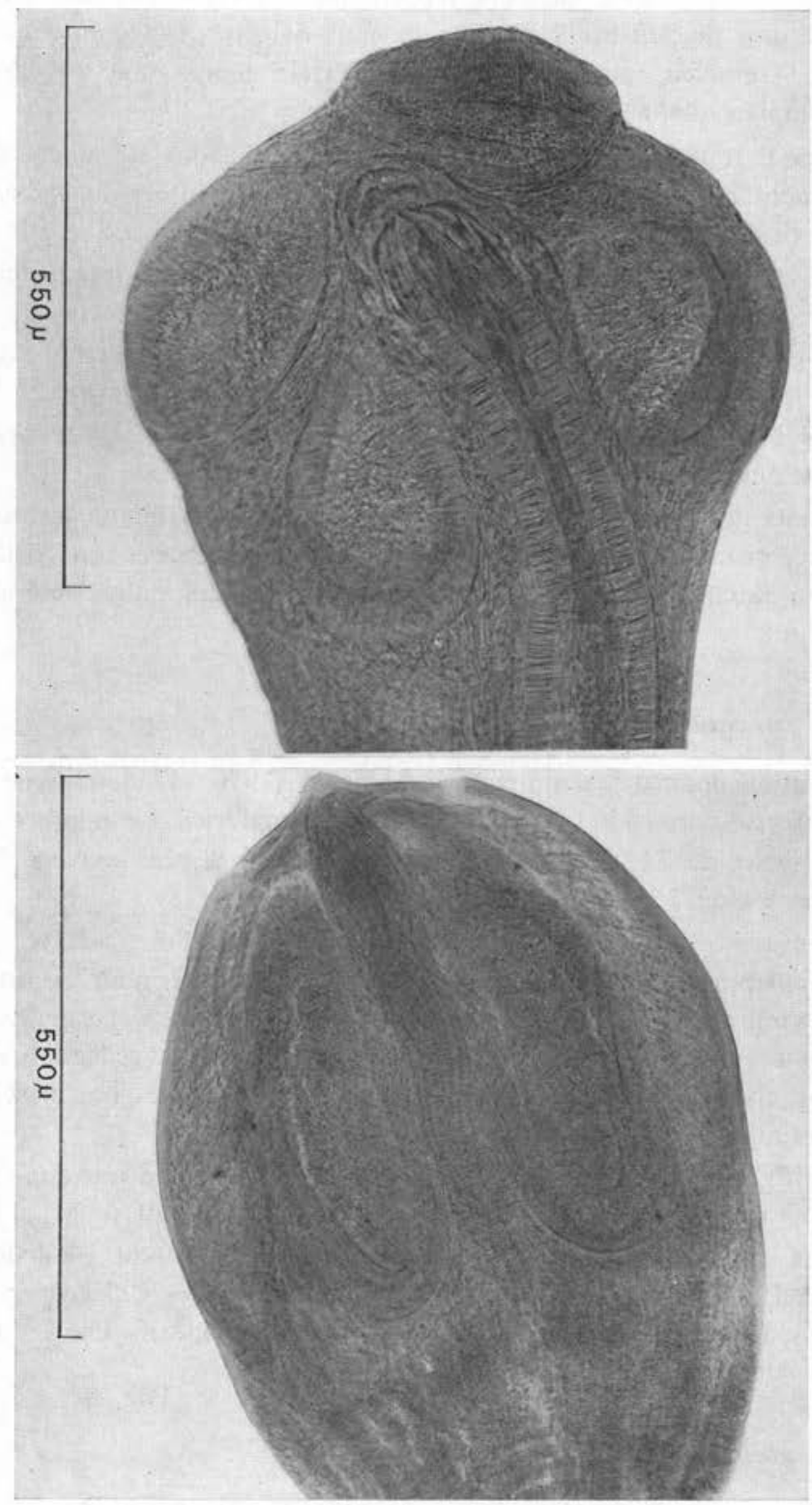

Planche 2. - Scolex photographiés au même grossissement. A : Ch. estavarensis; B : Ch. crassiscolex 
de vers, formés de plusieurs anneaux renfermant des œufs parfaitement mûrs, rencontrés dans le tube digestif de l'hôte. Les anneaux ovigères, isolés du reste du ver, sont très mobiles et semblent mener pendant un certain temps une vie libre jusqu'à la maturation complète des œufs.

Comme pour toutes les espèces sur lesquelles nous avons été amené à étudier des œufs parfaitement mûrs, en vue de la réalisation expérimentale du cycle, nous décrivons les œufs observés sur le vivant.

Les œufs de $H$. diaphana ont une enveloppe externe très fragile, ellipsoïde, qui mesure 44 sur $36 \mu$ en moyenne (fig. $6 \mathrm{D}$ ).

L'embryophore est formé par une paroi légèrement épaissie $(1 / 2 \mu)$. Il est de forme elliptique et présente deux petites pointes latérales. Il mesure 29 sur $24 \mu$ en moyenne. Une paroi fine double intérieurement l'embryophore. Entre ces deux enveloppes, on distingue toujours des sphères réfringentes.

Les crochets de l'embryon hexacanthe n'ont pas tous la même forme et la même taille. Les deux crochets centraux ont une lame plus recourbée que celle des paires latérales. Ils mesurent $11 \mu$ de longueur. Les crochets des paires latérales mesurent $9 \mu$ de longueur.

\section{Hymenolepis furcata (Stieda, 1862).}

La description donnée récemment par Vaucher (1970) d'individus de cette espèce récoltés chez Sorex correspond tout à fait à notre matériel. Le nombre des crochets observés peut varier de 22 à 28 . Nous en avons compté le plus souvent 27 . Leur longueur moyenne est de $25 \mu$ (pl. 1 A).

Les dimensions des œufs sont un peu supérieures à celles observées par Vaucher et se rapprochent plus de celles données par Zarnowski (1955) pour des individus trouvés chez «Crocidura leucodon ». Nous pensons d'ailleurs, comme l'a déjà souligné Vaucher, qu'une interversion d'hôtes s'est produite au cours de la rédaction du travail de Zarnowski, car nous n'avons nous même jamais trouvé cette espèce chez les Crocidures que nous étudions dans la région méditerranéenne.

Les œufs (fig. $6 \mathrm{E}$ ) ont une forme ellipsoïde et mesurent en moyenne 52 sur $39 \mu$. L'embryophore, peu épaissi, est sphérique et mesure de 29 à $34 \mu$ de diamètre (moyenne $31 \mu$ ). Les crochets de l'embryon hexacanthe mesurent $14 \mu$ de long. Nous avons cependant observé une différence dans la forme des différents crochets: les crochets internes des paires latérales sont nettement plus massifs que les autres (externes des paires latérales et paire centrale).

Hymenolepis globosoides (Soltys, 1954).

Cette espèce, assez rare, n'a été trouvée que chez les Sorex. Les caractères morphologiques et anatomiques de nos spécimens s'accordent parfaitement avec la redescription de l'espèce donnée par Vaucher (1970). Nous avons donné plus haut les principaux caractères qui permettent de différencier cette espèce de Hymenolepis fodientis. 
Hymenolepis jacutensis (Spassky et Morosov, 1959).

Ce Cyclophyllide, très rare, n'a été jusqu'ici signalé que dans trois localités européennes (Russie, Belgique, Allemagne). Ayant noté dans les diagnoses des différents auteurs (Brendow, 1969; Vaucher, 1970) des différences avec nos observations, nous donnons ci-dessous une description de l'espèce basée sur cinq individus mûrs.

Les individus mûrs peuvent mesurer jusqu'à $35 \mathrm{~mm}$ de longueur. Le scolex a en moyenne $420 \mu$ de diamètre sur $300 \mu$ de longueur. Les ventouses, ovalaires, mesu-
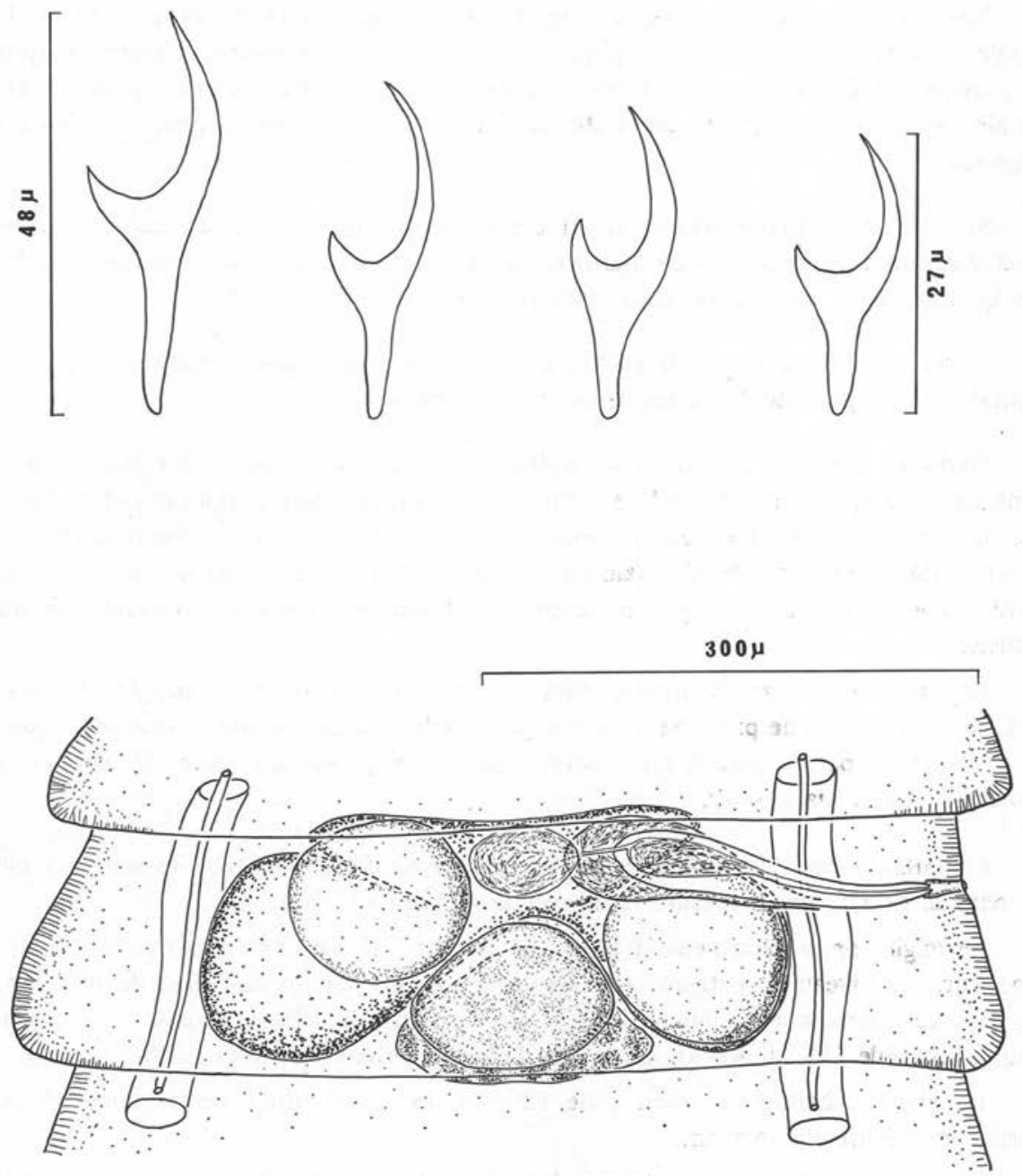

F1G. 4. - Hymenolepis jacutensis (Spassky et Morosov, 1959). A : Crochets d'un même rostre; B : Segment mûr, vue dorsale 
rent 180 sur $120 \mu$. Le ver possède un petit rostre de $120 \mu$ de longueur en moyenne, armé de 19 à 22 crochets non disposés en couronne. Les crochets d'un même scolex montrent une variabilité très importante dans leur longueur $(27$ à $48 \mu)$.

Par leur longueur et la dimension du scolex, nos exemplaires se rapprochent beaucoup de ceux observés par Brendow. En ce qui concerne l'armature du rostre, nos observations concordent tout à fait avec celles de Vaucher qui a également noté une grande variabilité dans la longueur des crochets et une disposition irrégulière de ces derniers sur le rostre (fig. $4 \mathrm{~A}$ ).

Nous avons compté jusqu'à 280 segments chez les individus les plus longs. Les proglottis sont toujours beaucoup plus larges que longs: les anneaux sexués mesurent en moyenne $800 \mu$ de largeur et $96 \mu$ de longueur. Les anneaux mûrs peuvent, chez certains spécimens, montrer une taille supérieure $(1,5 \mathrm{~mm}$ de largeur et $350 \mu$ de longueur).

Système osmo-RÉgulateur: Il est constitué par deux paires de canaux : les canaux ventraux mesurent $26 \mu$ de diamètre, les dorsaux sont beaucoup plus petits $(2,5 \mu)$. Les quatre canaux suivent un trajet assez rectiligne le long du strobile.

Système GÉNItAL (fig. $4 \mathrm{~B}$ et 5): Les pores génitaux sont unilatéraux. L'atrium génital s'ouvre près du bord antérieur des proglottis.

Organes MÂles: Les trois testicules occupent une position dorsale. Dans les anneaux sexués, ils sont disposés en triangle, un poral, deux antiporaux. Les testicules, de forme ovoïde, mesurent en moyenne 120 sur $90 \mu$. Les spermatozoïdes sont déversés dans une vésicule séminale externe de 96 sur $48 \mu$, située au milieu du segment. Celle-ci communique par un court canal déférent avec une vésicule séminale interne.

La poche du cirre, fusiforme, parallèle au bord antérieur du proglottis, mesure 225 sur $30 \mu$. Sa partie proximale est occupée par la vésicule séminale interne longue de $72 \mu$. Dans sa partie distale, on observe une région prostatique bien développée, entourant le canal éjaculateur.

Organes femelles: L'ovaire, lobé, occupe ventralement tout le segment entre les canaux excréteurs. Il mesure en moyenne $425 \mu$ de largeur.

Le vagin longe ventralement la poche du cirre. Il mesure en moyenne $192 \mu$ de longueur. Au niveau de l'atrium génital, il est entouré par un manchon de fibres musculaires qui forment un sphincter (fig. 5). A l'opposé, il débouche dans un réceptacle séminal, ovoïde ( 84 sur $48 \mu$ ), situé sous la partie proximale de la poche du cirre.

La glande vitellogène, ovoïde, de 120 sur $48 \mu$, est située ventralement dans la partie postérieure du segment.

L'utérus occupe tout le segment dans les derniers proglottis, repoussant latéralement les canaux osmorégulateurs. L'embryophore, ellipsoïde, mesure 30 sur $15 \mu$. 
Vaucher, dans sa description de l'espèce, donne comme longueur de la poche du cirre 154-165 $\mu$. Les longueurs que nous avons données se rapprochent nettement de celles signalées par Spassky et Morosov (1959) et Brendow (1969).

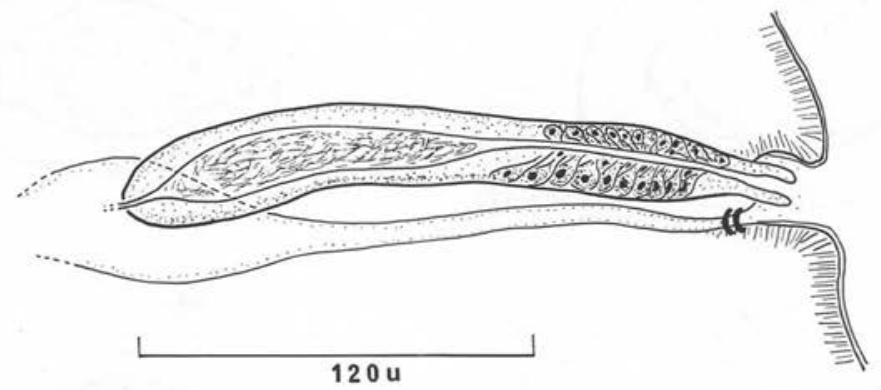

FIG. 5. - Hymenolepis jacutensis (Spassky et Morosov, 1959). Conduits sexuels terminaux. Coupe transversale.

Nous pensons que les spécimens étudiés par Vaucher étaient fortement contractés, ce qui expliquerait les dimensions réduites observées par cet auteur et la forme qu'il a donnée au segment sexué dans son schéma. Nous avons, en effet, souvent observé dans notre matériel des anneaux au moins huit fois plus larges que longs.

Hymenolepis schaldybini (Spassky, 1947).

Cette espèce est relativement abondante dans notre région. Vaucher a fait une remarquable mise au point systématique sur ce Cyclophyllide, démontrant notamment que la plupart des Hymenolepis scalaris décrits jusqu'ici chez les Musaraignes du genre Sorex étaient en réalité des Hymenolepis schaldybini (Spassky, 1947). Toutes les précisions anatomiques et les mensurations données par cet auteur concordent avec nos observations.

Ayant eu l'occasion de travailler sur un abondant matériel, nous donnons ci-dessous les mensurations des crochets rostraux et des œufs.

Nous avons compté sur tous les individus dix crochets mesurant en moyenne $38 \mu$ de longueur (pl. 1 B).

Les œufs, de forme ellipsoïde, mesurent en moyenne 77,5 sur 52,5 $\mu$. L'embryophore, ovoïde, mesure 43-45 $\mu$ sur 32,5-15 $\mu$ (moyenne 37,5 sur 27,5 $\mu$ ). Ce dernier, légèrement épaissi, présente deux petites pointes aux pôles de son plus grand axe (fig. $6 \mathrm{~F}$ ).

Les crochets de deux paires latérales mesurent $11 \mu$ de longueur, ceux de la paire centrale, plus grêles, mesurent $13 \mu$. 



FIG. 6. - Morphologie des œufs de Cyclophyllides de Soricinae. A: Hymenolepis fodientis; B : Hymenolepis magnirostellata; C : Choanotaenia estavarensis; D: Hymenolepis diaphana (1: crochet latéral; 2: crochet médian); E: Hymenolepis furcata (1: crochet médian; 2: crochet latéro-central); F : Hymenolepis schaldybini (1: crochet latéral ; 2 : crochet médian). 
Hymenolepis scutigera (Dujardin, 1845).

Nous n'avons trouvé cette espèce que dans très peu de stations. Lorsqu'elle est présente, sa densité et sa fréquence chez les Musaraignes sont cependant très élevées. La morphologie s'accorde totalement avec les descriptions faites par Dujardin (1845), Joyeux et Baer (1936) et plus récemment Vaucher (1970). Comme Vaucher, nous avons toujours compté 10 crochets de 32 à $36 \mu$ de longueur (pl. $1 \mathrm{C}$ ). N'ayant pas rencontré, au cours de nos recherches, des individus parfaitement mûrs, nous ne pouvons donner de précisions morphologiques sur les œufs.

Hymenolepis stefanskii (Zarnowski, 1954).

Nous avons rencontré assez souvent cette espèce chez les Musaraignes du genre Sorex. Les descriptions données jusqu'ici concordent très exactement avec nos observations.

Il est à remarquer que le nombre de crochets du scolex est assez variable entre deux nombres limites (14 et 22). Nous avons compté le plus souvent 15 crochets. Ceuxci mesurent $31 \mu$ de longueur en moyenne (pl. $1 \mathrm{D}$ ).

Un détail semble devoir être signalé en ce qui concerne les anneaux mûrs. En effet, nous avons toujours observé que le Cestode était terminé par un seul anneau ovigère. Au demeurant, les œufs contenus dans ce dernier segment ne sont pas embryonnés, leur maturation s'achevant dans les anneaux détachés du ver.

Les œufs, observés vivants, sont parfaitement sphériques et mesurent $10 \mu$ de diamètre en moyenne. Entourant l'embryophore, on observe souvent une enveloppe à l'aspect froissé.

L'embryophore, peu épaissi, mesure 27-25 $\mu$ sur 25-22 $\mu$ en moyenne (fig. 7 A).

\section{Hymenolepis prolifer (Villot, 1880).}

Cette espèce est très rare dans notre région. Nos exemplaires montrent tous les caractères observés par les auteurs qui ont donné de l'espèce une description très détaillée (Baer et Della Santa, 1960 ; Vaucher, 1970).

Les dimensions des embryophores de nos individus sont cependant un peu supérieures (31 sur $19 \mu$ en moyenne) à celles mesurées par ces derniers auteurs.

Choanotaenia crassiscolex (Von Linstow, 1898).

Cette espèce a été uniquement rencontrée chez les Musaraignes du genre Sorex, même dans les stations où il y a coexistence des populations de Soricidés des genres Neomys et Sorex. Il est important de souligner que cette spécificité stricte de Choanotaenia crassiscolex vis-à-vis de Sorex n'a été jusqu'ici observée que dans la région pyrénéenne. Dans toutes les stations européennes où l'espèce a été décrite, celle-ci parasite tous les genres de Soricidés: Sorex, Crocidura, Neomys. 
Les principaux caractères qui permettent de différencier cette espèce de Choanotaenia estavarensis sont résumés dans le tableau 3.

La planche 1 montre une photographie des crochets. La morphologie de l'œuf est donnée dans la figure $7 \mathrm{~B}$.

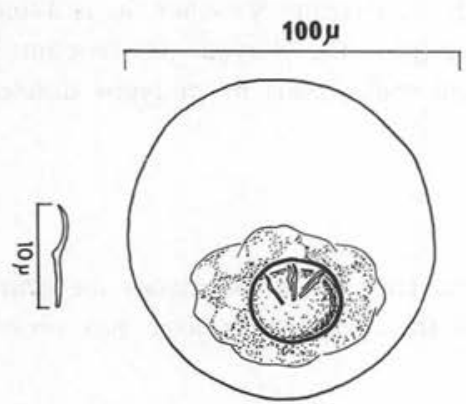

A

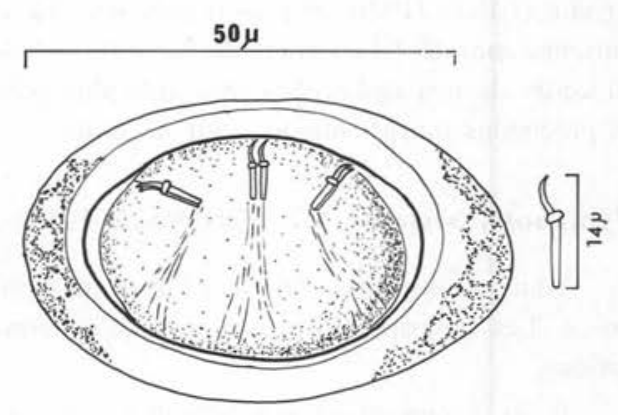

B

Fig. 7. - Morphologie des œufs de Cyclophyllides de Sorex. A : Hymenolepis stefanskii ; B : Choanotaenia crassiscolex

\section{Trématodes}

Brachylaemus fulvus Dujardin, 1843.

Cette espèce a été trouvée chez les Musaraignes du genre Sorex. Son microbiotope à l'intérieur de l'hôte est représenté par l'œsophage et la région du cardia. La présence de ce Digène dans l'intestin ne s'observe que chez les Musaraignes mortes depuis un certain temps et chez lesquelles les processus de décomposition intestinale entraînent une migration des parasites.

Lewis (1969) attribue les Brachylaemus récoltés dans l'œsophage des Soricidés à l'espèce Brachylaemus æsophagei Schaldybin, 1953. Cette espèce, placée en synonymie avec Brachylaemus fulvus Dujardin, 1843 par Zarnowski (1960), est considérée par contre par Lewis comme parfaitement distincte de l'espèce de Dujardin.

Nous ne partageons pas cette dernière position et considérons Brachylaemus asophagei comme synonyme de Brachylaemus fulvus, les caractères différentiels retenus par Lewis ne nous paraissant pas suffisamment fondés ; cet auteur base en effet sa distinction sur les caractères suivants :

- localisation différente des deux Digènes le long du tractus digestif: $B$. oesophagei serait une espèce œsophagienne, $B$. fulvus serait une espèce stomacale et intestinale ;

- différences dans les dimensions des organes.

La place du parasite à l'intérieur de l'hôte ne nous paraît pas pouvoir être considérée comme un caractère suffisamment valable puisque Brachylaemus fulvus a aussi 
été trouvé par la plupart des auteurs dans l'œsophage (Soltys, 1954 ; Prokopic, 1959 et nous-même). Nous pensons que Brachylaemus fulvus est un parasite essentiellement œsophagien, sa présence en-deçà de cet organe étant provoquée par la mort de l'hôte.

Le tableau 4 nous montre que les différences dans la taille des organes notées par Lewis sont minimes et ne peuvent être tenues pour significatives.

\section{Tableau 4}

Comparaison des mensurations de Brachylaemus fulvus Dujardin, 1843 et de Brachylaemus oesophagei Schaldybin, 1953

(Les dimensions autres que la longueur et la largeur du parasite sont donnés en $\mu$ ).

\begin{tabular}{|c|c|c|c|c|}
\hline & $\begin{array}{c}\text { Brachylaemus } \\
\text { fulvus } \\
\text { d'après } \\
\text { Dujardin } \\
(1843)\end{array}$ & $\begin{array}{l}\text { Brachylaemus } \\
\text { fulvus } \\
\text { d'après } \\
\text { Zarnoswski } \\
\text { (1960) }\end{array}$ & $\begin{array}{l}\text { Brachylaemus } \\
\text { oesophagei } \\
\text { d'après } \\
\text { Lewis } \\
(1969)\end{array}$ & $\begin{array}{c}\text { Brachylaemus } \\
\text { fulvus } \\
\text { présent } \\
\text { travail }\end{array}$ \\
\hline Longueur $m / m \ldots$ & 1,82 & $2,01-2,41$ & $1,350-2,120$ & $\begin{array}{c}1,850 \\
\text { en moyenne }\end{array}$ \\
\hline Largeur $\mathrm{m} / \mathrm{m} \quad \ldots$ & 0,70 & $0,48-0,80$ & $\overline{0,463-0,720}$ & $\begin{array}{c}0,624 \\
\text { en moyenne }\end{array}$ \\
\hline $\begin{array}{l}\begin{array}{l}\text { Ventouse } \\
\text { diamètre } \\
\text { verale } \\
\text { trans- }\end{array} \\
\text { versal } \ldots \ldots \ldots \ldots\end{array}$ & 130 & $180-240$ & $165-198$ & $\begin{array}{l}196 \\
\text { en moyenne }\end{array}$ \\
\hline 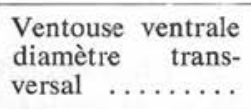 & 160 & $180-240$ & $190-230$ & $\begin{array}{l}236 \\
\text { en moyenne }\end{array}$ \\
\hline Pharynx $\ldots \ldots$. & 74 & $120-160$ & $110-140$ & $\begin{array}{c}130 \\
\text { en moyenne }\end{array}$ \\
\hline Testicules ...... & & $\begin{array}{c}180-230 \times \\
180-220\end{array}$ & $\begin{array}{c}140-180 \times \\
140-180\end{array}$ & $\begin{array}{l}149 \times 154 \\
\text { en moyenne }\end{array}$ \\
\hline Ovaire $\ldots \ldots \ldots$ & & $190 \times 210$ & $\begin{array}{c}130-170 \times \\
120-170\end{array}$ & $\begin{array}{l}120 \times 125 \\
\text { en moyenne }\end{array}$ \\
\hline Eufs $\ldots \ldots \ldots$ & $28-29$ & $26-30 \times$ & $\begin{array}{c}24-27 \times \\
16-18\end{array}$ & $\begin{array}{c}26 \times 14 \\
\text { en moyenne }\end{array}$ \\
\hline
\end{tabular}

Nephrotrema truncatum (Leuckart, 1842).

Cette espèce est fréquente chez les Sorex pyrénéens. Nous avons justifié plus haut la détermination spécifique de ce parasite rénal.

Pseudoleucochloridium soricis (Soltys, 1952).

Nous avons trouvé à plusieurs reprises, au niveau du côlon des Musaraignes, des Digènes le plus souvent immatures, dont tous les caractères morphologiques et anato- 
miques concordent très exactement avec les différentes descriptions (Soltys, 1952 et Pojmanska, 1961) de l'espèce Pseudoleucochloridium soricis.

Ces auteurs mentionnent pour cette espèce des taux d'infestation extrêmement faibles (de $0 \%$ à 2,6\% de Sorex araneus parasités suivant les années) que nous avons nous-même pu confirmer dans les Pyrénées. Par contre, nous avons observé, dans les stations qui nous ont livré le Digène adulte, des infestations massives (90\%) d'un Helicidé terrestre (Euomphalia strigella) par une métacercaire dont les caractères permettent d'affirmer qu'elle appartient indiscutablement au genre Pseudoleucochloridium.

Le déséquilibre entre le taux d'infestation du $2^{\mathrm{e}}$ hôte intermédiaire par la métacercaire que nous assimilons à celle de l'espèce $P$. soricis et celui des Musaraignes par le Digène adulte nous a conduit à entreprendre des recherches sur le cycle.

La dissection, $24 \mathrm{~h}$ après le repas infestant, de Musaraignes parasitées expérimentalement (Sorex minutus et Neomys fodiens) par les métacercaires mûres extraites des Helicidés ne nous a jamais livré ce parasite.

Nous pensons que cette espèce est en ráalité un Digène parasite d'Oiseau, mais dont certaines métacercaires, ingérées avec le Mollusque par les Musaraignes, peuvent évoluer chez ces derniers hôtes durant leur transit intestinal jusqu'au stade «Digène mûr », en un temps très court, inférieur à $24 \mathrm{~h}$, avant d'être éliminées. L'autopsie de Musaraignes, 5 à $6 \mathrm{~h}$ après l'infestation expérimentale, devrait nous permettre de trouver des Trématodes tout à fait semblables à ceux que nous avons rencontrés chez les Musaraignes infestées naturellement.

Cette étude nous permet de vérifier et de confirmer (Vaucher, 1970) la spécificité très stricte des Plathelminthes vis-à-vis de chaque genre de Soricidae. Dans les Pyrénées, nous avons pu voir que seul le Trématode Nephrotrema truncatum pouvait se rencontrer à la fois chez les Neomys et les Sorex.

Ces recherches mettent également en évidence l'originalité du parasitisme des Neomys fodiens pyrénéens. Ce dernier se caractérise en effet par la présence d'espèces très rares, certaines même non signalées jusqu'ici dans d'autres régions. Par contre, nous ne retrouvons pas chez cette Musaraigne les parasites qu'elle héberge habituellement partout ailleurs.

\section{Bibliographie}

BAER (J.-G.), 1931. - Un nouveau genre de Trématode provoquant des lésions dans le rein de la Taupe. Actes de la Soc. Helv. des Sciences Naturelles, 112, 337-338.

—, 1932. - Contribution à la faune helminthologique de Suisse. Rev. Suisse de Zool., 39, 1-56.

-, 1943. - Les Trématodes parasites de la Musaraigne d'eau Neomys fodiens (Schreb.). Bull. Soc. Neuchâtel. Sci. nat., 68, 33-84. 
Bychovskaya-Pavlovskaya (I. E.), Vysotzkaya (S. O.) et Kulakova (A. P.), 1970. - Trematodes of small mammals from transkarpathian region. Parasitologia, IV, (1), 26-34.

Combes (C.) et Jourdane (J.), 1969. - Pseudocephalotrema pyrenaica n. gen., n. sp. (Trematoda) parasite de Neomys fodiens (Pennant) dans les Pyrénées. Position taxonomique des genres Cephalotrema Baer, 1943 et Pseudocephalotrema n. gen. Vie et Milieu, 20 (1 C), 21-28.

Euzet (L.) et Jourdane (J.), 1968. - Helminthes parasites des Micromammifères des Pyrénées Orientales. I. Cestodes de Neomys fodiens (Schreber). Bull. Soc. Neuchât. Sc. Nat., 91, 31-42.

Genov (T.) et Dimitrova (E.), 1966. - On the helminthofauna of insectivorous mammals in Bulgaria. I. Trematoda and Cestoidea. Bull. centr. Helminthol. Lab. Kh., XI, 125-159.

KisIelewSKA (K.), 1961. - Circulation of tapeworms of Sorex araneus araneus L. in biocenosis of Bialowieza National Park. Acta parasitol. polon., IX (24), 331-369.

Lewis (J. W.), 1969. - Studies on the life history of Brachylaemus oesophagei Schaldybin, 1953 (Digenea: Brachylaemidae). J. Helminthol., XLII, 79-98.

PoJMANSKa (T.), 1961. - Investigations on the occurence and biology of trematodes of Sorex araneus araneus L. in Bialowieza National Park. Acta parasitol. polon., IX (23), 305-330.

Soltys (A.), 1952. - The helmints of common shrew (Sorex araneus L.) of the National Park of Bialowieza (Poland). Ann. Univ. Mariae Curie, Sklodowska, C, Pologne, VI (5), 165-209.

VAucher (C.) et UnKLer (P.), 1967. - Contribution à l'étude des Cestodes et des Trématodes parasites des micromammifères de Suisse. I. Bull. Soc. Neuchâtel. Sci. nat., 90, 161-184.

VAucher (C.), 1970. - Les Cestodes parasites des Soricidae d'Europe. Etude anatomique, révision taxonomique et biologie. Thèse Université de Neuchâtel (Suisse).

ZARNOWSKI (E.), 1960. - Parasitic worms of forest micromammalians (Rodentia and Insectivora) of the environment of Pulawy (district Lublin). II. Trematodes. Acta Parasitol. polon., VIII (8), 127-168. 